\title{
Diffusion tensor imaging: implications for brain disease
}

Victoria Singh-Curry, Richard E Roberts and Masud Husain

J. Neurol. Neurosurg. Psychiatry 2008;79;490-491

doi:10.1136/jnnp.2007.138388

Updated information and services can be found at:

http://jnnp.bmj.com/cgi/content/full/79/5/490-a

\section{These include:}

References This article cites 6 articles, 3 of which can be accessed free at: http://jnnp.bmj.com/cgi/content/full/79/5/490-a\#BIBL

\section{Rapid responses You can respond to this article at:} http://jnnp.bmj.com/cgi/eletter-submit/79/5/490-a

Email alerting Receive free email alerts when new articles cite this article - sign up in the box at service the top right corner of the article

Notes

To order reprints of this article go to:

http://journals.bmj.com/cgi/reprintform

To subscribe to Journal of Neurology, Neurosurgery, and Psychiatry go to:

http://journals.bmj.com/subscriptions/ 


\section{Should we accept the status quo? Time for new trials in status epilepticus}

\section{Margaret J Jackson}

Status epilepticus (SE), defined as a seizure or series of seizures without recovery between lasting more than $30 \mathrm{~min}$, takes a variety of forms, as illustrated in the report by Knake and colleagues $^{1}$ in this issue of $J$ Neurol Neurosurg Psychiatry (see page 588). In their paper, the response to intravenous levetiracetam of 16 patients with a total of 18 episodes of status epilepticus is described. The patients were all adults; the majority of episodes of SE were complex partial and symptomatic in aetiology, a case mix that might well be seen in an acute medical admissions unit. The duration of SE is not given, a pity particularly for the patients with convulsive SE where outcome is related to duration of seizure activity. All patients received intravenous benzodiazepine, usually lorazepam, before levetiracetam. Intravenous levetiracetam was given as a bolus dose of $250-1500 \mathrm{mg}$ and was associated with seizure cessation in 16/ 18 episodes; it was the second drug given in 10 episodes, the third in seven. Sedation was observed in two patients but no other side effects were noted. The study was

Correspondence to: Dr M J Jackson, Department of Neurology, Royal Victoria Infirmary, Queen Victoria Rd, Newcastle upon Tyne NE1 4LP, UK; margaret.jackson@ ncl.ac.uk retrospective, unblinded and observational, but the results suggest the use of intravenous levetiracetam in SE should be investigated further.

The incidence of SE is between 6.8 and $41 / 100000 /$ year in population based studies $^{2}$; prognosis depends predominantly on the aetiology. Early effective treatment is the goal. Despite being a relatively common neurological emergency, few randomised controlled trials of treatment in SE have been undertaken. A recent Cochrane review identified 11 studies of convulsive and non-convulsive status epilepticus. ${ }^{3}$ It concluded that intravenous lorazepam was superior to diazepam or phenytoin for initial treatment of established SE. When lorazepam fails in convulsive SE, current guidance recommends intravenous phenytoin, phenobarbital or fosphenytoin, all of which can cause hypotension, cardiac arrhythmia and hypoventilation. Approximately one-third of patients with convulsive SE fail to respond to secondline treatment and require admission to intensive care with the attendant risks of artificial ventilation. New, less toxic therapies for SE are needed.

Levetiracetam is one of the newer antiepileptic drugs. It has a broad spectrum of action being effective against both focal onset and primary generalised seizures. Since 2000, when it was licensed in Europe, the use of oral levetiracetam has increased swiftly; it has no significant interactions with other drugs, has a rapid titration schedule and is usually well tolerated. Significant side effects include behavioural change and unpredictable seizure exacerbation. The intravenous preparation became available in the UK in 2006; case reports of its use in convulsive and non-convulsive status epilepticus followed within months.

An alternative to intravenous levetiracetam or other standard therapies for SE is a drug that will be familiar to all neurologists-sodium valproate. Intravenous valproate has been compared with intravenous phenytoin in the treatment of SE in a randomised trial $^{4}$; the numbers were small but suggested valproate $30 \mathrm{mg} / \mathrm{kg}$ over 15 min was at least as effective as phenytoin. The time has come for another trial of treatment for SE. This will not be easy; issues around consent and funding need to be resolved and setting up a large multicentre study will be difficult. But it is a challenge we should not shirk if we want to try to improve treatment of a common and life threatening neurological condition.

\section{Competing interests: None.}

J Neurol Neurosurg Psychiatry 2008;79:490. doi:10.1136/jnnp.2007.135350

\section{REFERENCES}

1. Knake S, Gruener J, Hattemer K, et al. Intravenous levetiracetam in the treatment of benzodiazepine refractory status epilepticus. J Neurol Neurosurg Psychiatry 2008;79:588-9.

2. Chin RF, Neville BG, Scott RC. A systematic review of the epidemiology of status epilepticus. Eur J Neurol 2004;11:800-10.

3. Prasad K, et al. Anticonvulsant therapy for status epilepticus. Cochrane Database Syst Rev 2005(4):CD003723.

4. Misra UK, Kalita J, Patel R. Sodium valproate vs phenytoin in status epilepticus: a pilot study. Neurology 2006;67:340-2.

\section{Diffusion tensor imaging: implications for brain disease}

Victoria Singh-Curry, Richard E Roberts, Masud Husain

Institute of Neurology and Institute of Cognitive Neuroscience, UCL, and National Hospital for Neurology and Neurosurgery, London, UK

Correspondence to: Professor M Husain, Institute of Neurology and Institute of Cognitive Neuroscience, UCL, and National Hospital for Neurology and Neurosurgery, London WC1N 3BG, UK; m.husain@ion.ucl.ac.uk
One thing we have learned about brain disorders is that they do not usually respect boundaries between the cortex and underlying white matter. Damage to one has knock-on effects on the other, even in conditions which may preferentially affect white matter, such as multiple sclerosis.
But what are the implications of white matter damage? Could it be responsible for cognitive syndromes that are traditionally considered to result from cortical damage? The paper by Urbanski and colleagues ${ }^{1}$ in this issue of $J$ Neurol Neurosurg Psychiatry examines the possibility that disconnecting distributed cortical networks might play a critical role in the syndrome of unilateral neglect following stroke (see page 598).

These authors used diffusion tensor imaging (DTI), a new imaging method which is undergoing rapid development, ${ }^{2}$ to assess the integrity of white matter tracts connecting anterior and posterior 
A

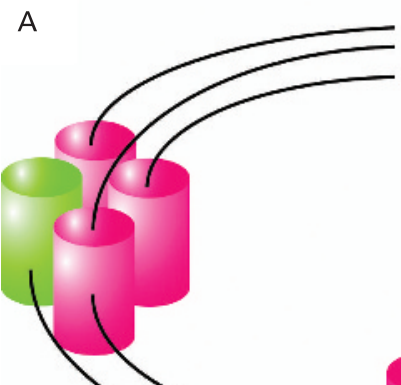

a
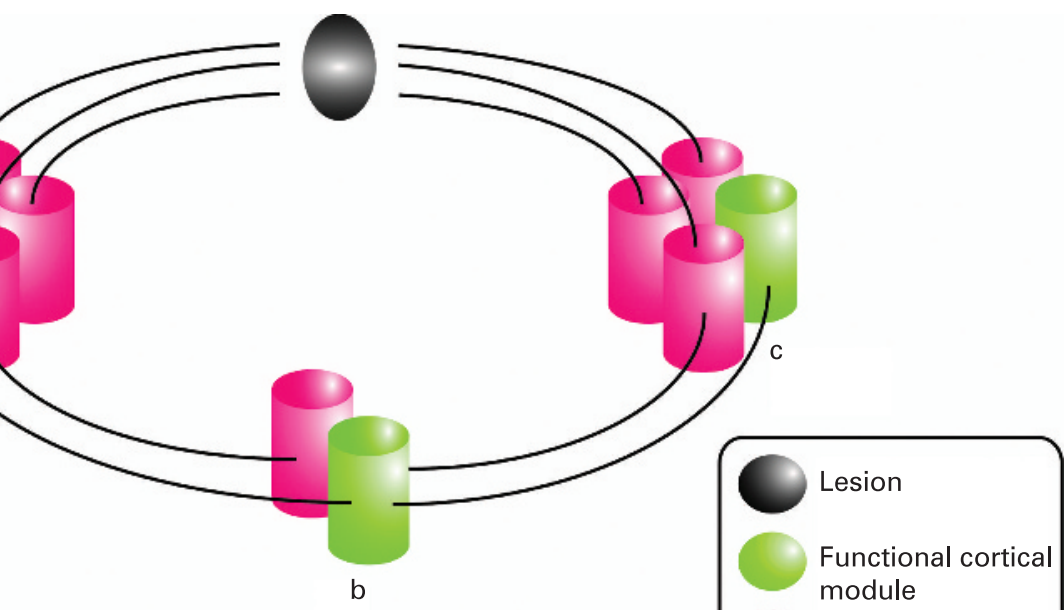

b

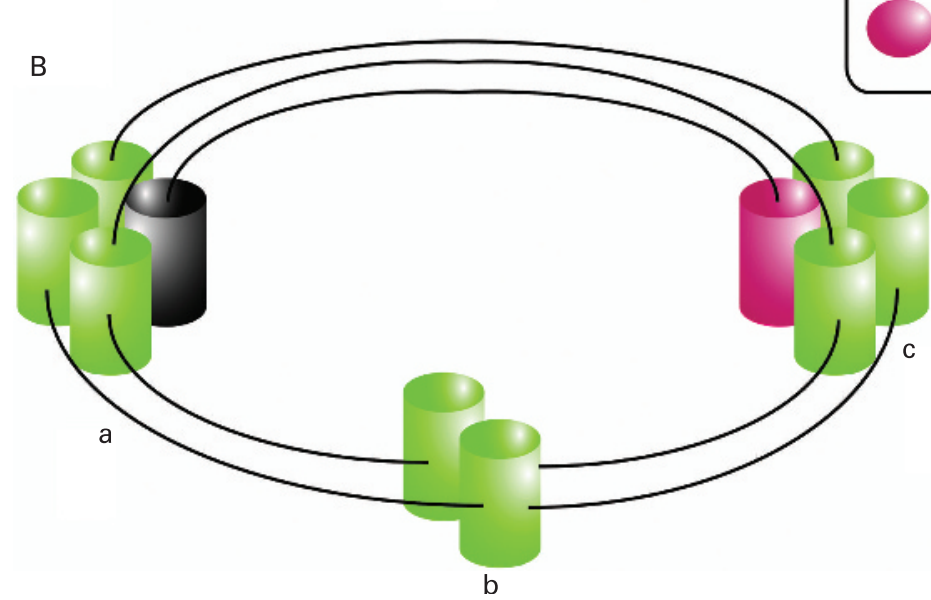

Figure 1 Network effects of disconnection. (A) Lesion of a tract connecting cortical modules leads to dysfunction (hypo- or hyper-activation) of some or all of the cortical areas to which the tract connects. Dysfunction of cortical modules $(a, c)$, caused by damage to white matter tracts connecting them, may also lead to altered activity in other, remote interconnected regions (b) even though the tracts leading to that region are intact. In this way, it is possible that a strategic lesion to a white matter tract can lead to dysfunction of an entire network. (B) A lesion directly affecting a single cortical module may affect other modules with which it is connected, but leads to less disintegration of function than a quantitatively similar lesion to a white matter tract.

regions of the right hemisphere. The results of their analysis revealed that damage to the inferior fronto-occipital fasciculus (IFOF), a tract that runs between the inferior frontal region to the occipital lobe, might be the crucial distinguishing factor between their patients with left-sided neglect and those without. The finding is both exciting and controversial, with wide implications far beyond the parishioners of the neglect syndrome.

Firstly, it suggests that selective damage to one tract might be sufficient to lead to a major neurological disorder. Urbanski and colleagues ${ }^{1}$ showed that two other large white matter tracts (the superior and inferior longitudinal fasciculi) may be entirely intact in their patients. Secondly, they provide a possible mechanism for understanding how neglect, which is most commonly associated with right parietal lesions, might follow from isolated lesions of the right frontal lobe. ${ }^{3}$ If the IFOF is lesioned, inferior frontal signals that bias attention in early visual areas may be compromised, without ever directly involving parietal cortex. The more general principle here is that disconnection may have long distance, remote effects far beyond the site of the lesion. Figure 1 illustrates how a small lesion has the potential to disconnect large scale cortical networks to produce complex cognitive deficits. Perfusion weighted MRI in patients with dysphasia or neglect shows that such subcortical lesions can produce widespread remote effects in intact cortical regions. ${ }^{4}$
The controversial part is the reliability of DTI. Recent studies in the rhesus monkey brain, using the much finer method of diffusion spectrum imaging as well as tracer studies, have found no evidence for the existence of an IFOF. ${ }^{5} 6$ This contrasts strikingly with results from classic human post-mortem studies, as well as more recent DTI investigations, which suggest the IFOF is indeed a distinct structure. It remains a possibility that the IFOF may be unique to humans, but far more work is required before we can accept this potentially remarkable conclusion. Moreover, the Urbanski study, ${ }^{1}$ although well executed, reports on a very small number of patients. It is likely that a complex syndrome such as neglect ${ }^{7}$ results from different combinations of direct cortical damage, white matter disconnection and indirect dysfunction of remote cortical regions in different patients. The same is also likely to be the case for other neurological disorders, where activity throughout a large scale network may be disrupted in many different ways.

In the next few years we are likely to see a multimodal imaging approach to complex brain disorders, combining structural and perfusion imaging with DTI to assess direct and indirect cortical effects, as well as disconnection within a brain network. This network perspective promises to be the next big step forward in understanding complex behavioural deficits at the systems level in a variety of conditions, including white matter diseases, stroke and degenerative disorders.

\section{Competing interests: None.}

J Neurol Neurosurg Psychiatry 2008;79:490-491. doi:10.1136/jnnp.2007.138388

\section{REFERENCES}

1. Urbanski $\mathbf{M}$, de Schotten M T, Rodrigo S, et al. Brain networks of spatial awareness: evidence from diffusion tensor imaging tractography. J Neurol Neurosurg Psychiatry 2008;79:598-601.

2. Alexander $\mathbf{A L}$, Lee JE, Lazar $\mathrm{M}$, et al. Diffusion tensor imaging of the brain. Neurotherapeutics 2007;4:316-29.

3. Husain M, Kennard C. Visual neglect associated with frontal lobe infarction. J Neurol 1996;243:652-7.

4. Hillis AE, Wityk RJ, Barker PB, et al. Subcortical aphasia and neglect in acute stroke: the role of cortical hypoperfusion. Brain 2002;125:1094-104.

5. Schmahmann JD, Pandya DN. Fibre pathways of the brain. New York: Oxford University Press, 2006.

6. Schmahmann JD, Pandya DN, Wang R, et al. Association fibre pathways of the brain: parallell observations from diffusion spectrum imaging and autoradiograpy. Brain 2007;130:630-53.

7. Husain M, Rorden C. Non-spatially lateralized mechanisms in hemispatial neglect. Nat Rev Neurosci 2003;4:26-36. 